\title{
Automated Hippocampal Segmentation by Regional Fluid Registration of Serial MRI: Validation and Application in Alzheimer's Disease
}

\author{
Rachael I. Scahill, William R. Crum, Nick C. Fox \\ Dementia Research Group, Institute of Neurology, 8-11 Queen Square, London, WC1N \\ 3BG United Kingdom \\ rscahill@dementia.ion.ucl.ac.uk
}

\begin{abstract}
Voxel-level three-dimensional fluid registration was used to propagate hippocampal segmentation from baseline MR scans onto follow-up scans. In this way automated measurements of hippocampal volume and volume changes were derived. The objective was to compare this technique with the current gold standard of manual segmentation. Serial measurements were performed on 15 normal controls and 12 Alzheimer's disease (AD) subjects. Hippocampal regions on the rigidly-registered repeat scans were generated both by manual segmentation and fluid registration. There was no significant difference between the repeat volumes generated by the two methods $(p<0.001)$, with the mean absolute volume difference being $4.6 \%$. Fluid registration is fully automated and has the potential to track longitudinal structural changes in the hippocampus with application to clinical trials in which large numbers of subjects require serial MR measurements.
\end{abstract}

\section{Introduction}

The hippocampus has been a particular focus for volumetric MR studies in AD due to its early involvement in the disease process, and cross-sectional studies have shown AD subjects with hippocampal volumes decreased by $20-40 \%$. Longitudinal studies reduce the problem of inter-individual variability and have shown greater rates of atrophy in $\mathrm{AD}$ subjects compared to controls ${ }^{1}$. However, the measurement errors produced by manual segmentation on serial scans may be of a similar magnitude to the changes expected within the structure, thereby reducing discriminating ability. The development of an automated technique to propagate a manually-generated baseline region onto a repeat scan could reduce this measurement error.

"Fluid Registration" is a non-linear matching algorithm which models the morphological changes within the brain as a compressible viscous fluid ${ }^{2-3}$. This study uses fluid registration to automate serial segmentation and quantification of volumetric change in the hippocampus. A comparison is made between this technique and manual segmentation by an experienced operator. 


\section{Materials and Methods}

Fifteen control subjects and 12 patients diagnosed with probable AD, according to the NINCDS-ADRDA criteria, underwent two MRI assessments with a mean $( \pm S D)$ interval of $536( \pm 380)$ days. Imaging was performed on a $1.5 \mathrm{~T}$ GE Signa Unit (General Electric Medical Systems, Milwaukee, WI) yielding 124 contiguous $1.5 \mathrm{~mm}$ thick slices, with in-plane resolution of $128 * 256$. All manual segmentation was performed using the MIDAS software package ${ }^{4}$. A rigid-body 9 degrees of freedom registration was performed on all repeat scans. An experienced operator, blind to subject condition, outlined the right hippocampus on the baseline and registered repeat scans. Following this a local fluid registration ${ }^{3}$ was applied to the manually-defined hippocampal region on the baseline scan. A repeat region was generated and the volume change calculated using the Jacobian values.

\section{Results}

The mean baseline hippocampal volumes were $2751( \pm 294) \mathrm{mm}^{3}$ in the controls and $2329( \pm 560) \mathrm{mm}^{3}$ in the AD subjects. There was close correlation between the repeat regions generated by manual segmentation and the fluid model $(\mathrm{r}=0.9, \mathrm{p}<0.001)$, with a mean absolute volume difference of $109( \pm 84) \mathrm{mm}^{3}, 4.6 \%$ of hippocampal volume.

\section{Discussion}

Manual segmentation remains the most widely used technique for the assessment of hippocampal volume in the study of AD. However, it is time-consuming and subject to observer error. Fluid registration is a fully-automated and unbiased technique for generating serial measurements which are consistent with manual segmentation. It could prove a useful tool with the growing application of serial imaging to the assessment of disease progression and monitoring of drug efficacy in clinical trials.

\section{References}

1. Jack CR, Petersen RC, Xu Y, O'Brien PC, Smith GE, Ivnik RJ, Tangalos EG and Kokmen E. Rate of medial temporal lobe atrophy in typical aging and Alzheimer's disease. Neurology 1998;51(4):993-9

2. Christensen GE, Rabbitt RD and Miller MI. Deformable templates using large deformation kinematics. IEEE Transactions in Image Processing 1998;5:1435-1447

3. Freeborough PA and Fox NC. Modeling brain deformation in Alzheimer's disease by fluid registration of serial 3D MR images. JCAT 1998;22(5):838-843

4. Freeborough PA, Fox NC and Kitney RI. Interactive algorithms for the segmentation and quantitation of 3-D MRI brain scans. Computer Methods and Programs in Biomedicine $1997 ; 53: 15-25$ 